\title{
THE DEVELOPMENT OF PERSONALITY PROFILING SYSTEMS ON THE ASSESSMENT OF INDIVIDUAL PERFORMANCE FOR THE BETTERMENT OF TRAINING PROGRAMME
}

\author{
Muhammad Nubli Abdul Wahab, Suriyakumar Sinnadurai, Mohd Azam Mohd Akhir, Rosasliza Samat \\ Center For Modern Languages And Human Sciences \\ University Malaysia Pahang, Kuantan 25000 Malaysia \\ e-mail mnubli@hotmail.com, suriya@ump.edu.my, azam@ump.edu.my
}

Tel: +60129683117 +6095492437

Training performance can be measured by a number of outputs resulting from completing individual activities in a training programme. A good training programme enhances knowledge, skills and attitudes which enables individuals to improve their performance. This paper has come up with a computerized heuristics system to assess individual's personality. It utiizes an expert system mode to assess individual's performance rather than utilizing manual assessments as currently being employed. The system is able to identify individual's personality strength and weaknesses and offer a detail evaluation and explanation on each individuals personality. The clarifications are important for each individual to understand their own behavior and how this can relate to their working performance. It is also useful to prevent assigning job tasks which does not fit with individual's traits and personalities. By utilizing the system, the user is able to explore the individual's personality and also in assisting the trainer in identifying suitable training programmes for their clients.

Key words: Personality profiing, heuristic systems, training programme

\section{Background}

Training performance can be measured by a number of outputs resulting from completing individual tasks in a training programme. A good training programme enhances knowledge, skills and attitudes which enables individuals to improve their performance. A good worker can workout multiple outputs within a very limited training time. The goal of training is for employees to master the knowledge, skill and behaviours emphasized in training programs and to be able to apply them in their day-to day tasks. Recently it has been acknowledged that to gain a competitive advantage, training has to involve more than just basic skills of development. That is, to use training to gain a competitive edge, thus training should be viewed broadly as a way to create intellectual capital. 
The personality profiling system is a guide in understanding personality styles as well as to increase effectiveness and satisfaction in a job. It is an assessment tool that will enable a person to develop a descriptive view of behavior tendencies and how they interact with others. All of us have developed behavioral patterns - distinct ways of thinking, feeling and acting. The central core of our patterns tends to remain stable because it reflects our individual identities. However, the demands of the work environment often require different responses that evolve into a work behavioral style. According to Brain Dynamic Global (2005) Personality is defined as the sum of mental, emotional, social and physical characteristics that distinguish one with another. Personality is a long term predisposition for behavior. It's strongly influenced by inherited endowment and works as predictor of pattern and behavior styles. Personality are enduring and relatively consistent characteristics of feeling, behavior and thinking.

The Personality Profiling System presents a plan to help a person understand oneself and others in a working environment. This profiling system enables a person to identify his/her working behavioral style, creates the motivational environment which is most conducive to bring about results and to minimize potential conflicts with others. This system is a self-administered and self-developed instrument. Conceived out of the needs expressed by individuals and work organizations, it collects and dis seminates information in a rapid an economical way. It is a scientifically researched communication tool which opens the door to greater understanding of oneself and others in order to build and maintain a sense of personal worth and self esteem in our personal and professional lives.

Each person has developed strengths and weaknes ses as a result of using preferences. Individual who are introvert tend to be serious, quiet, practical, orderly and logical. They can organize task, be decisive and follow through plans and goals. Hence Personality Profiling System can be used in identifying and analyzing elements for designing training programmes such as communication, motivation, teamwork, work style and leadership.

\section{The Design of the System}

This paper attempts to explain the design of the heuristic system that can gain access to individual's personality. It utilizes an expert system method to assess individual's performance rather than using manual assessments system that is usually used in psychometric test system. The system is able to identify individual' s personality, their strength and weaknes ses and it offers detail evaluation and explanation on each individual' s personality. The clarifications are important for each individual to understand their own behavior and how this can be related to their working performance. Understanding individual behavior is 
vital to ensure that the job assigned to them is appropriate to their strength. It is also useful to avoid assigning ajob which does not fit with individual's traits and personalities.

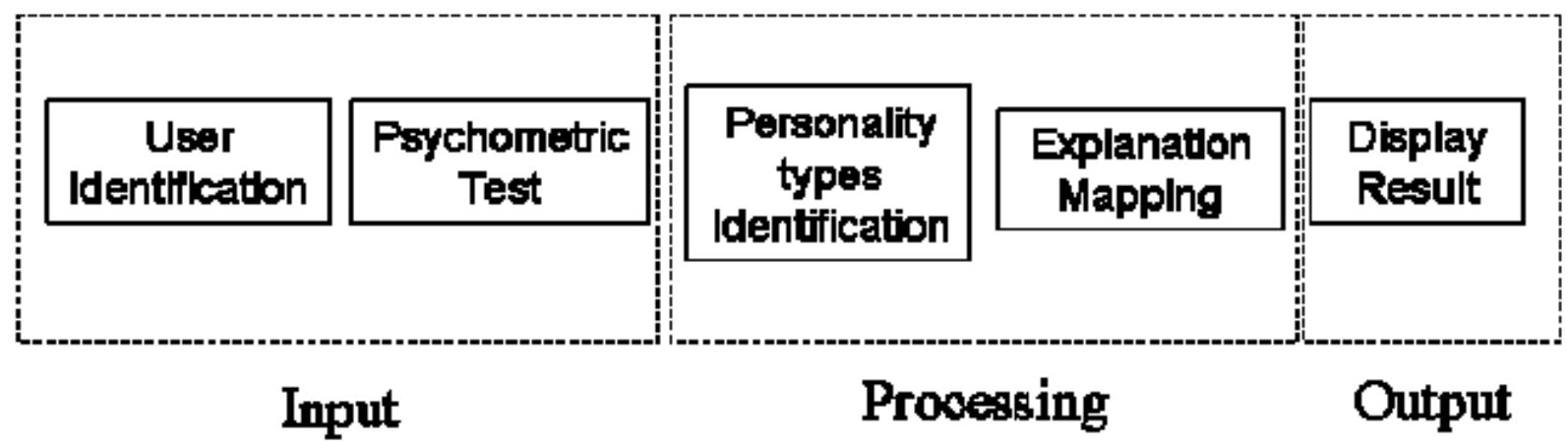

Figure 1: Basic design of the systems

Figure 1 describes the basic design of the system. It involves three major steps which is input phase, processing phase and the output phase. In input phase the user is required to key in their basic identification details which includes names, identity card number, gender and some personal information. These information is important to the system owner to identify individual's profile and also as an identification of the user of the system in the database. After that, the system will display brief guidelines about how to use the system. This will help users to understand the purpose of the test and how they can benefit from it. Before proceeding with the test, the system will require the consent of the user to agree with the terms of references in using the system.

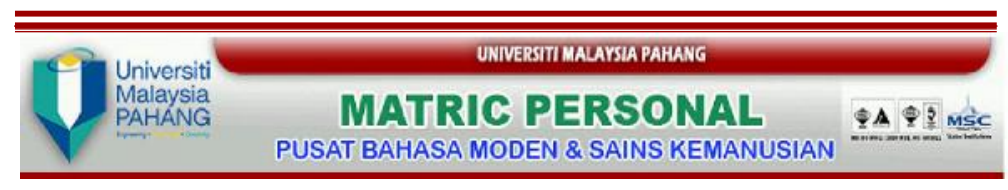

1. I am a confident and persuasive person

2. I have to be in the company of someone

3. I am ready to cooperate and assist someone

4. I am a result oriented person 
Figure 2: Screen shot of the personality test 
The second phase of the system involves analyzing the users psychometric information to match with specific types of personality. Figure 2 is the excerpt from a screenshot of the system which is used by the users to respond to the psychometric questions. From the responses, the system will analyze the answers and then categorized them into six categories. The categories, includes the dominance of personality in leadership and stability, extroverted, cooperation and values, discipline and result, visionary, and resoluteness. The responses given will be analyzed and interpreted based on six types of behavior. The system will perform the tasks automatically and will display the results to the users. The information or results obtained is vital to assist individuals in identifying their personality and also helping them in signing up appropriate training programmes.

Figure 3 is below is an example of the screen shot of the answers from the system. The explanation from the system will help users to identify their personality traits in detail together with the strength and weaknes ses of each personality. Understanding ones personality type will help the trainee and the trainer both to design and in drawing up suitable training programmes which fit the needs of organization.

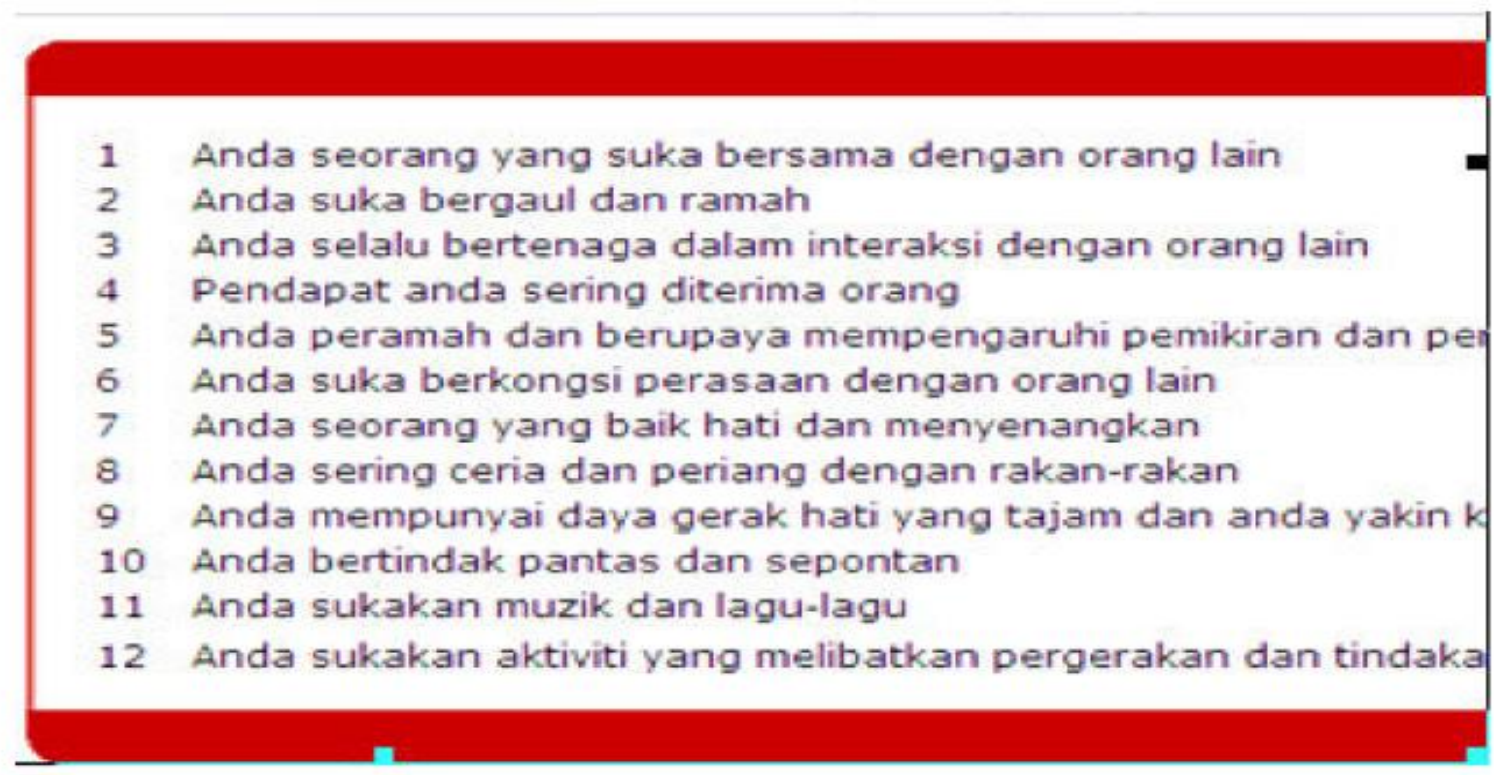

Figure 3: Screenshot of the personality explanation 


\section{Characteristics of the system}

This system was designed using a web based client server with a single database. The system is accessible to any user 24 hours worldwide. Users can log in to the systems by completing the required personel information. Web based system enables the systems owner to monitor the user of the system with its remote features. This can lock specific users from entering the system by blocking the internet protocol request from an invalid user. The web based system is also useful to the user because of the robustness of the system to the internet browser over the net. This will also give freedom to the user to enter the system when needed. Web based system also enables the system to be managed easily because the engine of the systems is part of the server and the manager can log onto the server to get access to the system. Besides, it also assists in maintaining the system and the design of the program is easily understood by other programmers

The system uses an user identification system. The system will authorize users to get access into the systems. Once the user's authentication is complete, they can use the system to identify their personality traits. The authorization and authentication of the system is done through identification of individual' $s$ identity card number. For that, the user needs to enter their identification number to gain access to the systems. Only he / she who know's the number and can get access to the system. The others cannot enter the system unless they know the specific individual' s identity card number as well as the owner of the number. This will provide safety features of the system in ensuring confidentiality of the information for each individual. 
SYSTEMS ARCHITECTURE

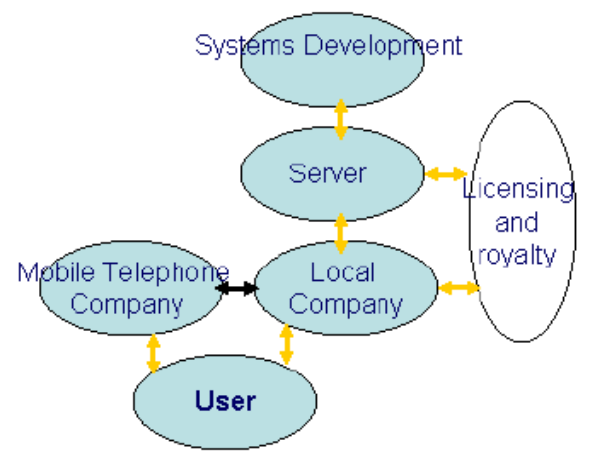

Figure 4: Systems Architecture

Figure 4: Systems Architecture 
Figure 4 provide explanations on the design of the system. One of the uniqueness of the systems is the involvement of local company as a mediator between users and the systems owner. The local company is also a mediator between system owner and mobile telephone company. The design is important because the local company acts as a service provider to the user. Term of agreement were agreed between the users and the system provided by the local company. The charging of using the systems is done through a mobile telephony service. Each user who uses the systems has to pay to get a specific access code. The code then will be used to enter into the system. The system will check the authenticity of the user by validating the assess code entered together with the identification card number. This design will assist the systems owner to maintain the system and help eliminate any hassle caused by the users of the system.

\section{Heuristic design of the systems}

One of the engine of the system is the matrix table used as a foundation to the heuristic system. The responses from the users will be transformed into a mean score for each cluster. Every cluster consists of 12 psychometric test items. The cluster also refers to specific personality types. From the responses, the system will calculate the total score of each cluster and it is divided by twelve to get the means score of the responses. The means score is than categorized into five extreme levels. The lowest is very week and the highest is very strong. Table 1 explains of the logic table used in the system. If the mean score from the user is 30 and below, the system will interpret the personality as very weak. If the means score of the responses in between 31 to 50 , the interpretation is weak. If the mean score range between 51 to 70 the answer is moderate. However if the response means score is more than 91 , it means that the personality is very strong.

\begin{tabular}{|l|l|}
\hline \multicolumn{1}{|c|}{ Meaning } & \multicolumn{1}{c|}{ Mean Score } \\
\hline Very Week & Below 10 \\
\hline VeryWeek & 11 to20 \\
\hline VeryWeek & 21 to 30 \\
\hline Weak & 31 to 40 \\
\hline Weak & 41 to 50 \\
\hline Moderate & 51 to 60 \\
\hline Moderate & 61 to 70 \\
\hline Strong & 71 to 80 \\
\hline Strong & 81 to 90 \\
\hline Very Strong & 91 to 100 \\
\hline VeryStrong & 101 to 110 \\
\hline VeryStrong & 111 to 120 \\
\hline
\end{tabular}

Table 1: Matrix of personality profiling in the system 
The main heuristic process in the system is the mapping process between mean score with the matrix table. The responses from the users will be matched with any range of the table and then will be interpreted based on the answer given in the matrix range. If the means score match with specific range, the interpretation will match with the matrix table of the explanation. These two tables will correspond together to provide explanation and guide the users.

\section{Benefit of the system}

The importance of this system is to help both the trainer and the trainee in identifying specific training programme which is suitable to them. To illustrate this, figure five, provides a detail explanation. Firstly, the training starts with identifying training objectives and suitable parameters. At the same time the user who wants to enroll on the programme needs to analyze his/her personality. The analysis of the personality will highlight and explain the types of personality that the trainee has as well as what are the suitable training techniques which is required for the training. Analysis and the determination of the types of personality together with the analysis of strength and weaknes ses of the users will help the trainer to realign training structures, training modules and training session which will benefit the trainee.

These information is also important to the trainer to set the parameters which is needed to measure the effectiveness of the programme. In the customization process, the trainer is not required to re align the training programme which is tailored to specific individuals. However there needs to be a minor modification of the training programme for the purpose of accommodating the needs and suitability of the trainer. Although the process is bit more detail, however, the programme is important in order to enhance the effectiveness of the training which usually subject to skepticism and also the consequences of the training programme to the organization. 


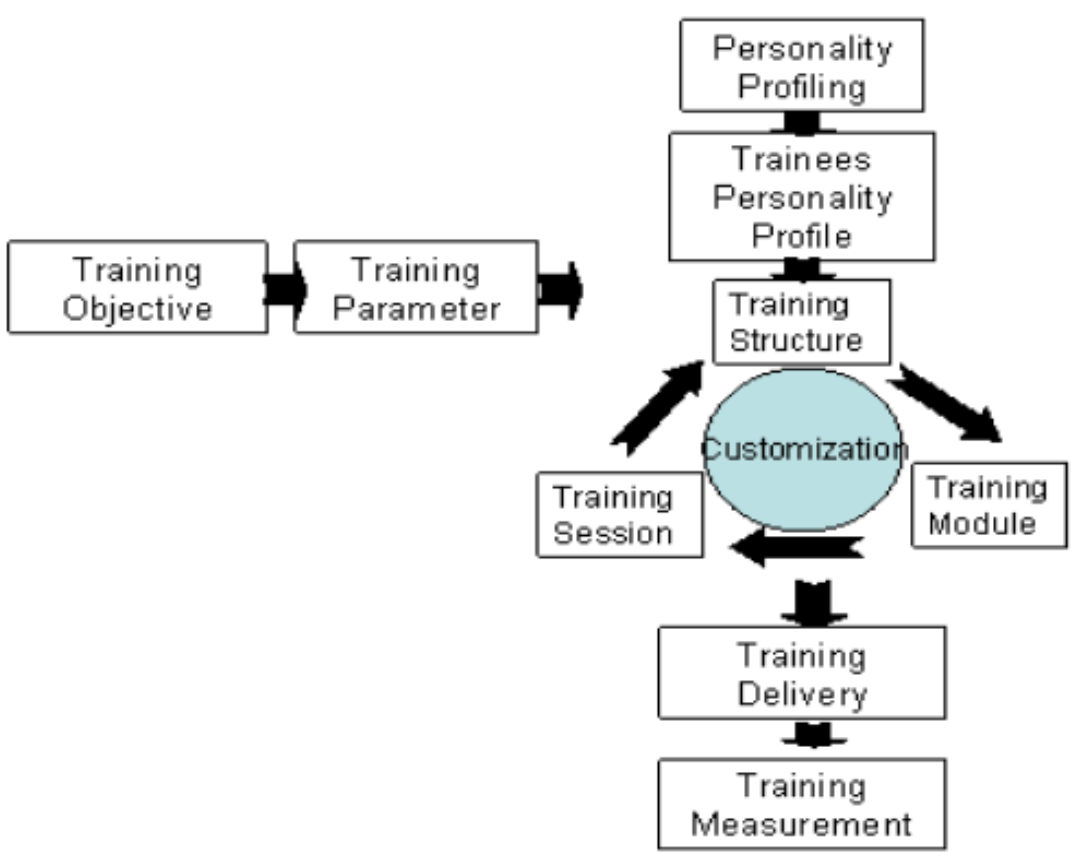

Figure 5: Uses of Personality Profiling the Designing Training Programme

The personality profiling system is used as a mechanism in improving training programme before any training can be delivered to the trainee. The technique is easy with the help of computer system. All then processes are done by the computer and the trainer only needs time to customize the training to match with the specific individual needs. Besides, these techniques helps to eliminate any errors when designing suitable training techniques especially when involves training in groups and tasks assigned in a team. This will ensure there is a better organized training program that meets the needs of the training

\section{Conclusion}

This paper describes the use of personality profiling system in designing a training programme. Besides , it explains about the design of information system used in personality profiling. These information is vital to the teacher, trainers, lecturers and those who are involved in training programmes. The system was designed to be hassle free and user friendly to help users use the systems effectively. What is most significant about the system is the users of the system will discover the easiness of identifying personality patterns and help them in designing quality training programmes. Although the system 
is not too comprehensive in its explanations, however, it can be used as a reference for personality profiling and can be upgraded to provide detailed explanations on each types of personality and also its remedies. Other researchers and educators can utilize the information provided by the system to assist in the understanding of the 
interpretation as well as to help individuals identify techniques in improving their personality. The uses of the system will help in delivering better quality training programs

\section{References}

A. Noe Raymong (2000), Employee Training \& Development, International edition, Singapore, McGraw Hill

Brain Dynamic Global (2005) The Credo Personality Profiling

C.E. Plott and J. Humphrey (1996), Preparing for 2020,"Training and Development (46-49)

David (2006), Information Systems Development : Methodologies, Techniques \& Tools, London : McGraw-Hill

Hershey (2006), Information Systems For Sustainable Development, London: Idea Group Inc

Steve (2005), Information systems : A Business Approach, Qld: John Wiley \& Sons Australia 